\title{
Perceptions dynamics about antiretroviral treatment among HIV patients in Bali
}

\author{
Anak Agung Sagung Sawitri ${ }^{1}$, I Nyoman Sutarsa ${ }^{2}$, Tuti Parwati Merati ${ }^{3}$, Made Bakta ${ }^{4}$, Dewa Nyoman \\ Wirawan $^{5}$ \\ 1,2,5 Department of Public Health and Preventive Medicine Faculty of Medicine Universitas Udayana, Indonesia \\ ${ }^{3,4}$ Department of Internal Medicine Faculty of Medicine Universitas Udayana/Sanglah General Hospital, Indonesia \\ ${ }^{2}$ Rural Clinical School, Medical School, the Australian National University, Australia
}

\begin{tabular}{l}
\hline \hline Article Info \\
\hline Article history: \\
Received Feb 27, 2021 \\
Revised May 22, 2021 \\
Accepted Jun 15, 2021
\end{tabular}

\section{Keywords:}

HIV

Outreach

Perception on ARV

Perception on clinics

Perception on health workers

\begin{abstract}
Perceptions of people living with HIV and AIDS (PLHIV) about antiretroviral treatment (ART) is crucial for improving adherence. This study aims to examine the dynamics of perceptions during the early phase of ART. A longitudinal study involving newly diagnosed PLHIV was conducted. Interviews were carried out at the time of HIV diagnosis and at three months after ART initiation. Data were analyzed by comparing proportion of good perception across the continuum of HIV diagnosis, ART initiation and threemonths follow-up, and were tested using Chi-square. From 170 PLHIV participated in the study, $81.76 \%$ had initiated ART and $73.4 \%$ remained on ART at three-months of follow-up. Several positive perception items were significantly decreased: ability to take ART at work and ability to continue treatment if experiencing side effects, effectiveness of ART, confidentiality, unwanted disclosure, and level of support from outreach workers. Ability to follow instruction from physician was significantly increased, and negative attitudes toward ART were decreased across the continuum. Adherence to ART is a continuous process, and is influenced by the dynamics perceptions among PLHIV. Understanding these dynamics is crucial to formulate strategies that can promote and maintain positive attitudes toward ART as well as living with HIV more broadly.
\end{abstract}

This is an open access article under the CC BY-SA license.

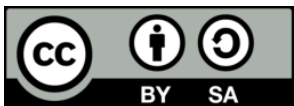

\section{Corresponding Author:}

Anak Agung Sagung Sawitri

Department of Public Health and Preventive Medicine

Faculty of Medicine, Udayana University

Jalan PB Sudirman Denpasar Bali 80223 Indonesia

Email: sagung_sawitri@unud.ac.id

\section{INTRODUCTION}

Antiretroviral therapy (ART) is effective in suppressing viral load, slowing down disease progression, and preventing premature death among people living with HIV and AIDS (PLHIV) [1], [2]. The global AIDS mortality has declined from 1.2 million to 770,000 between 2010 and 2019 due to significant increase in access to ART [3]. Several studies have identified that the main contributor of AIDS-related deaths is limited access to ART or delayed ART initiation [4], [5]. The benefits of ART can only be maximized when it is promptly administered (early ART initiation) with high level of compliance [6]. The global HIV prevention strategy has emphasized the implementation of comprehensive and sustainable services for PLHIV [7], [8], requiring PLHIV to be aware of their status early, get immediate care and support, initiate early medication [9], and maintain high level of adherence towards their medication [9], [10].

Indonesia has implemented the continuum of care and comprehensive health services for HIV (or locally called Layanan Komprehensif dan Berkelanjutan/LKB) since 2013. One pillar of this program is 
sustainable comprehensive HIV services packages, including: HIV testing and counseling services, management of sexually transmitted infections (STIs), TB-HIV collaboration, prevention of mother-to-child transmission, anti retroviral (ARV) treatment and treatment for opportunistic infections [11]. HIV counseling program is expected to facilitate the establishment of self-efficacy, positive perceptions about HIV treatment and care, and to reduce self-stigma. The primary objective is to increase the uptake of HIV testing and early ART initiation, as well as to improve adherence to ART [11].

Perceptions of PLHIV about ART are influenced by various factors including provider-related issues, stigma or fear of discrimination, and comprehensive understanding about benefits and side effects of ART. For instance, the acceptance of early ART initiation is influenced by the trust of PLHIV towards healthcare providers (e.g. doctors and clinic staff) where treatment is being offered [6], [12]-[14]. Trust of PLHIV toward health workers and facilities can be strengthen by promoting practices that maintain confidentiality and by providing non-discriminatory services or non-stigmatizing attitudes [13], [15]. Similarly, fear of side effects or treatment failure and considerations regarding harms and benefits of ART have been identified by many scholars as contributing factors for accepting or rejecting early ART initiation [12], [16]-[18]. PLHIV with good understanding about drug efficacy [19] and strong confidence in the ability to take medication regularly [12], [20] are more likely to accept ART. Furthermore, the long-term adherence towards ART is influenced positively by good interpersonal relationships with health providers in conducive clinical environments [12], adequate management of side effects [12], [21], sufficient availability of complex treatment modalities [12], and constructive experiences with social support systems [2].

Perceptions of PLHIV regarding ART, access to care and quality of health services may change over the course of the treatment. However, this is yet to be observed from existing studies where many are crosssectional in nature, capturing only a single event or phenomenon during the continuum of HIV treatment. We reported the changes in perceptions among PLHIV from a longitudinal study. This study investigated the dynamics of perceptions of PLHIV at three different points: at initial HIV diagnosis, at ART initiation, and at three months after ART initiation. Understanding the dynamics of perceptions among PLHIV throughout the HIV care continuum, especially over the course of anti retroviral (ARV) medication is crucial to better design strategies, including HIV counselling methods and contents as well as related prevention strategies for improving long-term adherence towards ART.

\section{RESEARCH METHOD}

This study was conducted at the WM Medika Clinic, an outpatient HIV and sexual health clinic at Kerti Praja Foundation (KPF) in Bali Province, Indonesia. KPF is a non-profit organization providing comprehensive HIV services which includes: HIV prevention through outreach and health promotion; HIV and ART counseling; diagnostic tests; treatment of sexually transmitted infections including HIV-AIDS; mitigation activities; and social support. These services are provided to the general population and key affected population groups such as female sex workers (FSW), men who have sex with men (MSM), people who inject drugs (PWID), and transgender women (waria).

The outreach activities at KPF are performed by outreach workers (OWs) to promote HIV prevention behaviors and to increase HIV testing coverage, especially among key populations. Clinic doctors and OWs are also trained as HIV counselors using the HIV counselling and testing training modules recommended by the Ministry of Health (MOH) [22]. HIV-positive clients are provided with general information about ART and HIV prevention, and are offered early ART initiation by OWs. Those who accept to initiate ART are referred to the doctor in the clinic for comprehensive health assessment prior to ART initiation and to provide clients with complete information regarding benefits, side effects and the importance of adherence to the treatment.

The study employed a longitudinal quantitative observational design with a cohort of newly-diagnosed HIV patients starting from 15 October 2015. These patients were followed until January 31, 2017. At the time of diagnosis, all PLHIV aged $\geq 18$ years-old were offered ART and were asked to participate in the study. Written consent was obtained from those who agreed to participate in the study. They were interviewed by the research team to explore their perceptions about ART and the quality of services they had received at the time of HIV diagnosis, at ART initiation, and at three months after the initiation. For the purpose of this study, a maximum of three months was given to PLHIV either to accept or to decline the treatment [23]. We collected the data at three occasions referred here as T1, T2, and T3. T1 was a point when patients were firstly diagnosed with HIV; T2 was a point of ART initiation; and T3 was a point of three months of follow-up after ART initiation. The last date of ART initiation was on September 24, 2016. Subsequent observations after this date were made only to PLHIV who accepted and initiated ART. The first interview was conducted at the beginning of the study (T1, n=170), and the second interview was conducted at three-months after ART initiation (T3, $\mathrm{n}=102$ ) to re-evaluate their perceptions about the treatment and the quality of health services they had received. No interview was performed at the ART initiation (T2). Perceptions of PLHIV who finally accepted and 
initiated ART $(\mathrm{T} 2, \mathrm{n}=139)$ were extrapolated from the first interview data (by excluding data of those who declined the treatment, $n=31$ ). The last date of the three-month follow-up was on November 26, 2016.

We used a structured questionnaire to assess perceptions of PLHIV. This questionnaire was pretested among PLHIV and the revised version was used in this study. Data were collected through structured interviews using this questionnaire. The interviews were performed by trained enumerators which included 17 OWs and three research assistants. At T1, interviews were conducted by OWs for the convenient of the patients because OWs usually accompanied the patients when they received their HIV diagnosis. At T3, interviews were performed by research assistants.

We assessed perceptions of PLHIV in seven domains; each domain consists of 3-7 items. These domains (or sub-perception categories) included: perceptions of being able to take medication (seven items), positive attitudes toward ART (six items), negative perceptions toward ART (four items), perceptions towards doctors (four items), perceptions towards OWs (four items) and perceptions about the clinic (three items). Each item was rated on a scale of 1 to 4 , with ' 1 ' as being 'unsure' and '4' as being very confident. A cumulative score was generated for each sub-perception category. Because of the data were not normally distributed, we used the median value to categorize perception into 'good perception' ( $\geq$ median) and 'poor perception' ( $<$ median). The acceptance of ART at T2 was defined as 'accept' when PLHIV have commenced ART and as 'not accept' if they refused the treatment or did not provided a response to the ART offer within three months of their diagnosis. We also explored socio-demographic characteristics of PLHIV.

Quantitative analysis was conducted by categorizing perception items based on the median value; and was compared using the median test between two-three times of measurement (T1, T2 and T3). Each sub-item of perceptions was compared using Chi-square test, between T1, T2 and T3. Socio-demographic characteristics were also compared in three times of T1, T2, and T3. All analyses were performed using STATA 12 (StataCorp LLC, Texas, USA).

The study was approved by the Human Research Ethics Committee, Faculty of Medicine Udayana University-Sanglah General Hospital on October 5, 2015 (Protocol Number: 1649/UN.14.2/Litbang/2015). The extension of the study period was approved by the committee on October 5, 2016 (Protocol Number 484/UN.14.2/KEP2016).

\section{RESULTS}

A total of 189 newly diagnosed PLHIV were offered to initiate ART and were invited to participate in the study. Of $170(89.9 \%)$ who agreed to participate in the study, a total of $139(81 \%)$ accepted ART. Three months after ART initiation as many as $102(73.3 \%)$ PLHIV remained on ART. A total of 170 respondents were successfully interviewed at T1 (at the time of HIV diagnosis) and 102 respondents at T3 (three months after ART initiation).

\subsection{Socio-demographic characteristics}

Table 1 depicts socio-demographic characteristics of PLHIV at the time of HIV diagnosis (T1) and those who initiated ARV treatment (T2). In terms of socio-demographic characteristics, we found no significant difference between the two groups (T1 vs T2). The majority of PLHIV (both in T1 and T2) were male, with an average age of 30 years, highly educated, employed, middle income, unmarried, and the majority were MSM.

Table 1. Socio-demographic characteristics of PLHIV

\begin{tabular}{|c|c|c|c|c|c|c|c|c|c|}
\hline \multicolumn{2}{|c|}{ Characteristics } & \multicolumn{2}{|c|}{$\mathrm{T} 1(\mathrm{n}=170)$} & \multicolumn{2}{|c|}{$\mathrm{T} 2(\mathrm{n}=139)$} & \multicolumn{2}{|c|}{ T3 $(n=102)$} & \multirow{2}{*}{$\begin{array}{c}\mathrm{p} \\
\mathrm{T} 1-\mathrm{T} 2\end{array}$} & \multirow{2}{*}{$\begin{array}{c}\mathrm{p} \\
\mathrm{T} 2-\mathrm{T} 3\end{array}$} \\
\hline & & $\mathrm{n}$ & $\%$ & $\mathrm{n}$ & $\%$ & $\mathrm{n}$ & $\%$ & & \\
\hline \multirow[t]{2}{*}{ Sex } & Female & 55 & 32.35 & 43 & 30.9 & 30 & 29.41 & 0.782 & 0.804 \\
\hline & Male & 115 & 67.65 & 96 & 69.1 & 72 & 70.59 & 0.658 & 0.804 \\
\hline \multirow[t]{3}{*}{ Educational level } & Illiterate-elementary school & 35 & 20.59 & 25 & 17.98 & 18 & 17.65 & 0.563 & 0.538 \\
\hline & Junior high school & 25 & 14.71 & 22 & 15.82 & 14 & 13.73 & 0.790 & 0.653 \\
\hline & High school and university & 110 & 64.71 & 92 & 66.19 & 70 & 68.63 & 0.784 & 0.690 \\
\hline \multirow[t]{2}{*}{ Employment status } & Unemployed & 23 & 13.53 & 19 & 14.00 & 15 & 14.70 & 0.730 & 0.878 \\
\hline & Employed & 147 & 86.70 & 120 & 86.00 & 87 & 85.29 & 0.858 & 0.876 \\
\hline \multirow[t]{3}{*}{ Marital status } & Not married & 97 & 57.06 & 83 & 59.70 & 62 & 60.78 & 0.640 & 0.866 \\
\hline & Married & 28 & 16.47 & 23 & 16.55 & 16 & 15.69 & 0.959 & 0.858 \\
\hline & Widowed/divorced & 45 & 26.47 & 33 & 23.75 & 24 & 23.53 & 0.583 & 0.968 \\
\hline \multirow[t]{3}{*}{ Risk group } & Heterosexual & 27 & 15.88 & 25 & 17.96 & 16 & 15.69 & 0.627 & 0.643 \\
\hline & FSW & 45 & 26.47 & 33 & 23.74 & 21 & 20.59 & 0.583 & 0.562 \\
\hline & Homosexual and bisexual & 98 & 57.65 & 81 & 58.30 & 53 & 51.97 & 0.909 & 0.329 \\
\hline Age & Median (IQR) (year) & \multicolumn{2}{|c|}{$30.1(10.9)$} & \multicolumn{2}{|c|}{$29.7(8.9)$} & \multicolumn{2}{|c|}{$30.0(11.3)$} & 0.933 & 0.943 \\
\hline Salary & Median (IQR) (million, IDR) & \multicolumn{2}{|c|}{$2.0(1.5)$} & \multicolumn{2}{|c|}{$2.0(1.5)$} & \multicolumn{2}{|c|}{$2(1.5)$} & 1.000 & 1.000 \\
\hline Work duration & Median (IQR) (year) & \multicolumn{2}{|c|}{$2.0(3)$} & \multicolumn{2}{|c|}{$2.0(3)$} & \multicolumn{2}{|c|}{$2.0(3)$} & 1.000 & 1.000 \\
\hline
\end{tabular}




\subsection{Perceptions of PLHIV at the beginning of the study (T1), ART initiation (T2) and three months after ART initiation (T3)}

Table 2 present the dynamics of perceptions among PLHIV about ART and the quality of HIVrelated services they had received at three different times. The median values of positive perceptions toward ART, negative attitudes toward ARV and perceptions toward the clinic were significantly differed between T1 (HIV diagnosis, $n=170$ ) and T2 (ART initiation, $n=139$ ). It signals that perceptions about ARV drug and the quality of HIV-related services, particularly toward the clinic, have significantly influenced the decisions of PLHIV to initiate early ART.

Table 2. Proportion of good perceptions about ART and HIV-related services received by PLHIV at the time of HIV diagnosis (T1), ART initiation (T2) and three months after ART initiation (T3)

\begin{tabular}{|c|c|c|c|c|c|c|}
\hline Perception items (\% of "good perception”) & $\mathrm{T} 1 \mathrm{n}=170$ & $\mathrm{~T} 2 \mathrm{n}=139$ & T3 $n=102$ & $\begin{array}{c}\mathrm{p} \\
(\mathrm{T} 1-\mathrm{T} 2)\end{array}$ & $\begin{array}{c}\mathrm{p} \\
(\mathrm{T} 1-\mathrm{T} 3)\end{array}$ & $\begin{array}{c}\mathrm{p} \\
\text { (T2-T3) }\end{array}$ \\
\hline Perception of ability to take ARV (median, IQR) & $23(5)$ & $23(6)$ & $21(3)$ & 0.115 & 0.000 & 0.006 \\
\hline Collect medication monthly & 94.70 & 96.40 & 97.06 & 0.475 & 0.359 & 0.778 \\
\hline Follow physician's instructions & 90.59 & 88.80 & 98.04 & 0.605 & $0.017^{*}$ & $0.006^{*}$ \\
\hline Consume medication on schedule & 95.30 & 96.40 & 94.12 & 0.632 & 0.670 & 0.402 \\
\hline Consume medication at workplace & 92.36 & 94.96 & 78.43 & 0.355 & $0.001 *$ & $0.000 *$ \\
\hline Consume medication during travel & 92.94 & 94.97 & 95.10 & 0.460 & 0.476 & 0.963 \\
\hline Consume medication during illness & 90.59 & 93.52 & 94.12 & 0.348 & 0.301 & 0.849 \\
\hline Continue medication when experiencing side effects & 95.30 & 98.56 & 92.16 & 0.107 & 0.287 & $0.014 *$ \\
\hline Positive beliefs of ARV (median, IQR) & $18(4.3)$ & $19(5)$ & $17(5.0)$ & 0.000 & 0.263 & 0.000 \\
\hline ARV can improve overall health & 91.76 & 95.69 & 93.14 & 0.162 & 0.680 & 0.386 \\
\hline ARV increases the longer life expectancy & 90.00 & 92.09 & 90.19 & 0.524 & 0.960 & 0.605 \\
\hline ARV increases the possibility to work normally & 91.76 & 94.25 & 91.17 & 0.397 & 0.867 & 0.356 \\
\hline ARV prevents progression to AIDS & 89.42 & 92.09 & 92.15 & 0.423 & 0.458 & 0.986 \\
\hline ARV reduces the number of virus in the body & 90.00 & 92.08 & 83.33 & 0.526 & 0.107 & $0.036^{*}$ \\
\hline ARV increases immunity & 90.00 & 93.53 & 85.29 & 0.266 & 0.243 & $0.035^{*}$ \\
\hline Negative beliefs of ARV (median, IQR) & $10(4.0)$ & $10(3)$ & $6(1)$ & 0.010 & 0.000 & 0.000 \\
\hline ARV increases possibility of hospitalization & 68.82 & 70.50 & 2.94 & 0.750 & $0.000 *$ & $0.000 *$ \\
\hline ARV causes severe side effects & 32.35 & 30.93 & 73.52 & 0.790 & $0.000 *$ & $0.000 *$ \\
\hline ARV increases risk for tuberculosis, rhinitis, diarrhea & 39.42 & 39.56 & 4.90 & 0.980 & $0.000 *$ & $0.000 *$ \\
\hline ARV causes unwanted disclosure of HIV status & 74.12 & 74.82 & 8.82 & 0.888 & $0.000 *$ & $0.000 *$ \\
\hline Perception to Physician (median, IQR) & $13(4.0)$ & $13(4.0)$ & $13(4.0)$ & 0.514 & 0.826 & 0.432 \\
\hline Physicians suggest the best option & 98.14 & 98.56 & 97.06 & 0.775 & 0.562 & 0.419 \\
\hline Physicians provide good service & 98.24 & 97.84 & 98.04 & 0.780 & 0.905 & 0.914 \\
\hline Physicians maintain confidentiality & 95.29 & 96.41 & 92.16 & 0.626 & 0.288 & 0.149 \\
\hline Physicians are supportive of treatment & 99.42 & 99.28 & 100.00 & 0.878 & 0.441 & 0.391 \\
\hline Perception to Outreach Workers (median, IQR) & $16(4.0)$ & $16(4.0)$ & $15(5.0)$ & 0.387 & 0.003 & 0.033 \\
\hline OWs maintain confidentiality & 97.65 & 97.84 & 87.25 & 0.911 & $0.001^{*}$ & $0.001 *$ \\
\hline OWs treat PLHIV equally with other patients & 95.29 & 96.40 & 94.12 & 0.629 & 0.673 & 0.149 \\
\hline OWs are prepared to assist with ARV issues & 98.82 & 99.28 & 97.05 & 0.682 & 0.294 & 0.181 \\
\hline OWs are prepared to support other problems & 91.17 & 90.65 & 69.60 & 0.874 & $0.000 *$ & $0.000 *$ \\
\hline OWs remind PLHIV to take their medication & 96.47 & 96.40 & 90.19 & 0.974 & 0.033 & $0.049 *$ \\
\hline Perception toward KPF Clinics (median, IQR) & $9(3.0)$ & $10(3.0)$ & $9(2.0)$ & 0.000 & 0.093 & 0.000 \\
\hline $\mathrm{KPF}$ is appropriate for ART & 97.65 & 98.56 & 97.06 & 0.564 & 0.766 & 0.420 \\
\hline KPF maintains confidentiality of PLHIV & 95.88 & 97.13 & 95.1 & 0.555 & 0.762 & 0.411 \\
\hline KPF is well-equipped with necessary facilities & 95.29 & 94.96 & 92.16 & 0.893 & 0.288 & 0.374 \\
\hline
\end{tabular}

* Significant at $\mathrm{p}<0.05, \mathrm{~T} 1=$ PLHIV agree to participate in study, T2 = PLHIV who initiate ART, T3 = PLHIV who remained on ART three months after ART initiation

In addition, perceptions of PLHIV were varied between those who were offered ART (T1, n=170) and those who remained on ART after three-months of follow-up (T3, n=102). Similarly, perceptions of PLHIV who accepted and initiated ART $(\mathrm{T} 2, \mathrm{n}=139)$ and those who remained on ART after three months of follow-up (T3, n=102) were also significantly differed. These findings suggest that adherence towards ART might be influenced by perceptions of PLHIV about ART and the quality of HIV-related services they had received.

In terms of the perceived ability to take ART, we found three items that might influence adherence towards ART which include: (1) 'ability to follow physician's instruction'; (2) 'ability to consume medication at the workplace'; and (3) 'ability to continue consuming medication when experiencing side effects'. PLHIV who remained on ART after three months of follow-up (T3) have higher confidence about their ability to follow physician's instructions. However, their perceived ability to be able to consume ART at workplace and to continue taking ART while experiencing side effects were significantly lower compared to at the time of HIV diagnosis (T1) or at ART initiation (T2).

We identified that positive beliefs about ART (i.e. 'ARV reduces number of virus in the body' and 
'ARV increases immunity') among PLHIV who accepted and initiated ART (T2) were significantly higher than those who remained on ART after three months of follow-up (T3). Similarly, we found that negative perceptions among PLHIV about ART were decreased significantly from T1 to T3. Perceptions of PLHIV about the physician and clinic remained relatively constant across the three points (T1, T2 and T3). However, we observed that three out of five items of perceptions toward OWs were decreased significantly across the three points. These items included: 'OWs maintain confidentiality'; 'OWs are prepared to support PLHIV with other problems', and 'OWs remind PLHIV to take their medication'.

\section{DISCUSSION}

The current study is the first to examine the dynamics of perceptions of newly diagnosed HIV patients until three months of follow-up after ART initiation. Previous studies fail to capture such dynamics because they only provide a 'snapshot' of perceptions using cross-sectional analysis [25]. Our study addresses this knowledge gap through longitudinal study with serial analysis at the time of HIV diagnosis, at ART initiation, and at three months after ART initiation.

Our study found that newly diagnosed HIV patients and PLHIV who accepted and initiated ART have high level of positive attitudes about their ability to take medication, effectiveness of ART, health providers and the quality of HIV-related services they had received. However, after three months on ART their perceptions had changed. Our serial analysis revealed small fluctuations of perceptions at the beginning of HIV diagnosis, at ART initiation and three months after ART initiation. However, it is sufficient to signal that adherence to ART is a continuous process. Positive experiences and supportive environment are likely to enable PLHIV to comply with their medication plan.

At the beginning of the treatment, we found that PLHIV have strong perceptions about their ability to take a lifetime medication and to manage side effects of the treatment. These strong perceptions are contributed by their understanding about ART provision [26], [27], adverse effects of the treatment [28], [29], and their self-worth and self-efficacy [12], [20], [30]. However, after on ART for three months, these positive attitudes were decreased significantly, especially with regard to their ability to take medication regularly at workplace and their ability to continue medication if they experience side effects. These findings are consistent with other study which found that PLHIV face difficulties to take pills daily and to manage their medication schedule with other daily activities [21], mainly if they were afraid of stigma or unwanted disclosure [12], [31], [32]. While the introduction of single dose drugs (oral or injectable) [33], [34] could address such issue, these modalities are currently not available in our study location. However, we observed an increase perceived ability to follow instructions from physician after being on ART for three months. Arguably, this might be attributed by supportive and conducive environment from health providers at the clinic.

Our study identified that negative perceptions about ART were high following the HIV diagnosis, especially the perception that 'ART will cause unwanted disclosure of HIV status'. This indicates that disclosure or stigma remains a major concern of many newly diagnosed HIV patients [12], [18], [32], [35][37]. It can be argued that many 'at risk' individuals might share the same perception about HIV testing and unwanted disclosure leading to low coverage of HIV counselling and testing [36]. However, three months after ART initiation, our analysis found that these negative perceptions were significantly decreased to $<10 \%$. This finding is promising and is consistent with findings from other studies [13], [38]. Positive experiences throughout the treatment alongside non-discriminatory care relationships between PLHIV and health providers have enabled trust formation that their privacy and confidentiality are protected.

In addition, almost one-third of our research participants were anxious about negative effects of ART at the time of HIV diagnosis. It is quite common for newly diagnosed HIV patients to feel worried about side effects, long-term risks and treatment failure [28]. Negative perceptions toward certain medication could outweigh the actual tolerability to such medication [29] leading to higher risk of experiencing side effects. Our serial analysis showed a two-fold increase of negative perceptions towards side effects after being on ART for three months, which could influence their adherence to ART. It is important to note that $13.5 \%$ of PLHIV in our study discontinued their medication due to side effects of the treatment, as also reported in various studies [39], [40]. Even after three-months of follow-up, we found that 27.6\% of PLHIV continued to experience side effects from the treatment and they remained on ART regardless of the side effects. Maintaining positive attitudes toward ART by leveraging positive care relationships between PLHIV and health providers, conducive and non-discriminatory health services, and delivering positive messages through formal and informal communication strategies [36] is crucial to improve treatment adherence.

Perceptions of PLHIV toward physicians, the clinic and the quality of HIV-related care they had received were relatively positive and stable throughout the continuum of HIV diagnosis, ART initiation and three-month follow-up, except for the outreach workers. Many studies have investigated the roles of 
physician in improving self-efficacy and adherence towards ART [20], [37], [41]-[43]; as well as the roles of clinic (or other healthcare facilities) in improving compliance towards HIV medication [12]-[14]. While the roles of health provider in general have been discussed in the literature [44], to the best of our knowledge, there is no existing study ever discusses the roles of outreach workers in improving self-efficacy of PLHIV and ART adherence. Our study addresses this gap by providing the crucial roles and positions of outreach workers, the backbone of HIV prevention, care and treatment in Indonesia, in improving self-efficacy of PLHIV and adherence to ART. Additionally, outreach workers are the primary contact for key population groups who require HIV testing, and for newly diagnosed HIV patients who decided to start medication. Our study found that perceptions of PLHIV toward outreach workers were highly positive at the time of HIV diagnosis; however, it was significantly decreased over time particularly with regard to confidentiality issues and the level of support provided by outreach workers. In addition, this finding supports the important aspect of counseling to communicate deeply about ARV drug with PLHIV to increase the successful of counseling provided by outreach workers [24].

The substantial decrease of PLHIV throughout the continum of HIV diagnosis (T1, $n=170$ ), ART initiation (T2, n=139), and three months follow-up (T3, n=102) suggests that adherence to ART is a continuous process which is influenced by the dynamics of perceptions of PLHIV about ART and the quality of HIV-related services they had received. Promoting positive perceptions during HIV counseling and testing is just as crucial as maintaining positive attitudes and experiences at ART initiation and throughout the treatment [23], [36], [45]. The three-months follow-up is particularly critical when positive perceptions have started to decrease while at the same time some negative attitudes have started to develop.

The present study has several limitations. First, the instrument used to capture perceptions of PLHIV was developed based on the health belief model (HBM) framework. It requires comprehension and prior knowledge about HIV and/or ART to be able to respond accurately to the questions/statements, which in some cases can be very challenging for PLHIV. Second, the dynamics of perceptions were only collected from PLHIV who remained on ART after three months of follow-up. They are more likely to have more positive attitudes and relatively stable perceptions over the continuum of HIV diagnosis, ART initiation and follow-up. Third, at the time of HIV diagnosis, PLHIV were interviewed by their outreach workers for the convinient of the participants. However, this strategy might create social desirability effect for which participants provide answers/responses to please the interviewer. Lastly, we recruited our study participants using a consecutive selection process which is vulnerable to selection bias, and therefore reducing the generalisability of the findings.

\section{CONCLUSION}

Adherence to ART is a continuous process influenced by the dynamics of perceptions among PLHIV related to their HIV diagnosis, ART and the quality of HIV-related care they had received from healthcare facilities. Understanding these dynamics is crucial to design, formulate and implement strategies that can promote and maintain positive attitudes toward ART as well as living with HIV more broadly, particularly among key population groups who might experience social exclusion from the society. Further studies should be done to encounter several limitations in this study, such as simplified the questionnaire, include PLHIV who has withdrawal from the ART, and randomly select the sample.

\section{ACKNOWLEDGEMENTS}

We would like to thank all research participants and all outreach workers who have provided valuable time and information for this study. We also thank all research assistants: Ika Farmani, Made Karma, and Estrada who have assisted in the data collection and the longitudinal observation. We would like to extend our gratitude to the Director of Kerti Praja Foundation for allowing the study to be conducted in the foundation's clinic.

\section{REFERENCES}

[1] WHO, CDC, and The Global Fund, "HIV drug resistance report 2017," 2017. doi: 10.1039/c4dt03267e.

[2] CDC, "Trends in HIV Infection Stage 3," Atlanta, 2017. [Online]. Available: https://www.cdc.gov/hiv/library/slideSets/index.html.

[3] UNAIDS, “UNAIDS DATA 2019," Switzerland, 2019. [Online]. Available: https://www.unaids.org/sites/default/files/media_asset/2019-UNAIDS-data_en.pdf.

[4] J. M. Granich, R., Gupta, S., Hersh, B., Williams, B., Montaner, J., Young, B., and Zuniga, "Trends in AIDS Deaths, New Infections and ART Coverage in the Top 30 Countries with the Highest AIDS Mortality Burden; 1990-2013," PLoS One, vol. 10, no. 7, 2015, doi: 10.1371/journal.pone.0131353. 
[5] D. Nabukalu et al., "Population-level adult mortality following the expansion of antiretroviral therapy in Rakai, Uganda," Popul. Stud. (NY)., vol. 74, no. 1, pp. 93-102, Jan. 2020, doi: 10.1080/00324728.2019.1595099.

[6] G. H. Friedland, "HIV medication adherence. The intersection of biomedical, behavioral, and social science research and clinical practice," J. Acquir. Immune Defic. Syndr., vol. 43, no. 1, pp. S3-S9, 2006, doi: 10.1097/01.qai.0000248333.44449.ea.

[7] M. J. Mugavero, K. R. Amico, T. Horn, and M. a. Thompson, "The state of engagement in HIV care in the United States: From cascade to continuum to control," Clin. Infect. Dis., vol. 57, no. 8, pp. 1164-1171, 2013, doi: 10.1093/cid/cit420.

[8] S. MacCarthy et al., "The HIV care cascade: Models, measures and moving forward," J. Int. AIDS Soc., vol. 18, no. 1, pp. 1-5, 2015, doi: 10.7448/IAS.18.1.19395.

[9] M. a. Thompson et al., "Guidelines for improving entry into and retention in care and antiretroviral adherence for persons with HIV: Evidence-based recommendations from an international association of physicians in AIDS care panel," Ann. Intern. Med., vol. 156, no. 11, pp. 817-833, 2012, doi: 10.7326/0003-4819-156-11-20120605000419. Guidelines.

[10] E. M. Gardner, M. P. McLees, J. F. Steiner, C. Del Rio, and W. J. Burman, "The spectrum of engagement in HIV care and its relevance to test-and-treat strategies for prevention of HIV infection," Clin. Infect. Dis., vol. 52, no. 6, pp. 793-800, 2011, doi: 10.1093/cid/ciq243.

[11] Indonesian Ministry of Health, Guidance of Comprehensive Services of HIV-AIDS and STI in Correctional Institutions/Prison (In Indonesia: Pedoman Layanan Komprehensif HIV-AIDS and IMS di Lapas, Rutan dan Bapas). 2012.

[12] M. Gwadz et al., "HIV-Infected Individuals Who Delay, Decline, or Discontinue Antiretroviral Therapy: Comparing Clinic- and Peer-Recruited Cohorts," Front. Public Heal., vol. 2, no. July, pp. 1-14, 2014, doi: 10.3389/fpubh.2014.00081.

[13] H. L. Surratt, C. L. O'Grady, S. P. Kurtz, M. E. Buttram, and M. A. Levi-Minzi, "HIV Testing and Engagement in Care among Highly Vulnerable Female Sex Workers: Implications for Treatment as Prevention Models," J. Health Care Poor Underserved, vol. 25, no. 3, pp. 1360-1378, 2014, doi: 10.1353/hpu.2014.0113.

[14] M. B. Laws et al., "Treatment acceptance and adherence in HIV disease: Patient identity and the perceived impact of physician-patient communication," Patient Prefer. Adherence, vol. 6, pp. 893-903, 2012, doi: 10.2147/PPA.S36912.

[15] A. Geter, A. R. Herron, and M. Y. Sutton, "HIV-Related Stigma by Healthcare Providers in the United States: A Systematic Review," AIDS Patient Care STDS, vol. 32, no. 10, pp. 418-424, 2018, doi: 10.1089/apc.2018.0114.

[16] K. T. L. Sy et al., "Predictors of antiretroviral therapy initiation in eThekwini (Durban), South Africa: Findings from a prospective cohort study," PLoS One, vol. 16, no. 2 February 2021, pp. 1-15, 2021, doi: 10.1371/journal.pone.0246744.

[17] K. Phiri et al., "Community and health system factors associated with antiretroviral therapy initiation among men and women in Malawi: a mixed methods study exploring gender-specific barriers to care," Int. Health, vol. 13, no. 3, pp. 253-261, 2021, doi: 10.1093/inthealth/ihaa041.

[18] S. Ahmed et al., "Why do people living with HIV not initiate treatment? A systematic review of qualitative evidence from low- and middle-income countries," Soc. Sci. Med., vol. 213, pp. 72-84, 2018, doi: 10.1016/j.socscimed.2018.05.048.

[19] J. Valeri, "Knowledge of HIV-AIDS a dominant factor of antiretroviral therapeutic adherence in women with HIVAIDS Correspondence: The United Nation global report on the," vol. 34, no. 2, pp. 129-137, 2016, doi: 10.18051/UnivMed.2016.v35.129-137.

[20] P. K. REACHOUT Centre Trust, MEWA, The Omari Project, North Star Alliance, ISHTAR, "Reaching key populations and improving their access to HIV services in Kenya Research Report," 2015. [Online]. Available: https://www.hivgaps.org/.../Research-report-Reaching-key-populations-and-improvin...\%0A\%0A.

[21] S. A. Iacob, D. G. Iacob, and G. Jugulete, "Improving the adherence to antiretroviral therapy, a difficult but essential task for a successful HIV treatment-clinical points of view and practical considerations," Front. Pharmacol., vol. 8, no. NOV, 2017, doi: 10.3389/fphar.2017.00831.

[22] Indonesian Ministry of Health, National guidelines for HIV and AIDS testing and counseling (In Indonesia: Pedoman nasional tes dan konseling HIV dan AIDS). 2014. [Online]. Available: https://docplayer.info/29575731Pedoman-nasional-tes-dan-konseling-hiv-dan-aids.html

[23] T. Mabuto, S. Charalambous, and C. J. Hoffmann, "Effective interpersonal health communication for linkage to care after HIV diagnosis in south africa," J. Acquir. Immune Defic. Syndr., vol. 74, pp. S23-S28, 2017, doi: 10.1097/QAI.0000000000001205.

[24] A. A. S. Sawitri, I. N. Sutarsa, K. T. P. Merati, I. M. Bakta, and D. N. Wirawan, "Why Counseling Intervention Fails to Improve Compliance towards Antiretroviral Therapy: Findings from a Mixed-Methods Study among People Living with HIV in Bali Province, Indonesia," Infect. Dis. Rep., vol. 13, no. 1, pp. 136-147, Feb. 2021, doi: 10.3390/idr13010015.

[25] R. M. Grimes and D. E. Grimes, "Readiness: The state of the science (or the Lack Thereof)," Curr. HIV/AIDS Rep., vol. 7, no. 4, pp. 245-252, 2010, doi: 10.1007/s11904-010-0056-2.

[26] C. J. Cohen, J. L. Meyers, and K. L. Davis, "Association between daily antiretroviral pill burden and treatment adherence, hospitalisation risk, and other healthcare utilisation and costs in a US medicaid population with HIV," BMJ Open, vol. 3, no. 8, pp. e003028-, 2013, doi: 10.1136/bmjopen-2013-003028.

[27] D. Therkelsen, "Challenges To Antiretroviral Adherence Among MSM And LGBTI Living With HIV in Kampala, 
Uganda," Durban South Africa, 2015. [Online]. Available: https://www.heard.org.za/publications-post/challengesto-antiretroviral-adherence-among-msm-and-lgbti-living-with-hiv-in-kampala-uganda/.

[28] T. G. Mthembu and B. Van Wyk, "Patients' knowledge and beliefs about antiretroviral treatment and factors associated with adherence in Mpumalanga Province, South Africa," Heal. SA Gesondheid, vol. 19, no. 1, pp. 1-7, 2014, doi: 10.4102/hsag.v19i1.718.

[29] R. Horne, S. C. E. Chapman, R. Parham, N. Freemantle, A. Forbes, and V. Cooper, "Understanding patients' adherence-related Beliefs about Medicines prescribed for long-term conditions: A meta-analytic review of the Necessity-Concerns Framework," PLoS One, vol. 8, no. 12, 2013, doi: 10.1371/journal.pone.0080633.

[30] H. Sylvain and P. Delmas, "Readiness in HIV treatment adherence: A matter of confidence. An exploratory study," Open AIDS J., vol. 5, no. 2, pp. 119-124, 2011, doi: 10.2174/1874613601105010119.

[31] E. R. N. Weaver, M. Pane, T. Wandra, C. Windiyaningsih, Herlina, and G. Samaan, "Factors that influence adherence to antiretroviral treatment in an urban population, Jakarta, Indonesia," PLoS One, vol. 9, no. 9, pp. 1-7, 2014, doi: 10.1371/journal.pone.0107543.

[32] C. O. Izugbara and E. Wekesa, "Beliefs and practices about antiretroviral medication: A study of poor urban Kenyans living with HIV/AIDS," Sociol. Heal. Illn., vol. 33, no. 6, pp. 869-883, 2011, doi: 10.1111/j.14679566.2010.01328.x.

[33] M. Barnhart, "Long-Acting HIV treatment and prevention: Closer to the threshold," Glob. Heal. Sci. Pract., vol. 5, no. 2, pp. 182-187, 2017, doi: 10.9745/GHSP-D-17-00206.

[34] S. Carillon et al., "Perspectives of injectable long acting antiretroviral therapies for HIV treatment or prevention: understanding potential users' ambivalences," AIDS Care - Psychol. Socio-Medical Asp. AIDS/HIV, vol. 32, no. sup2, pp. 155-161, 2020, doi: 10.1080/09540121.2020.1742869.

[35] S. Willard and A. Angelino, "The need for sociocultural awareness to maximize treatment acceptance and adherence in individuals Initiating HIV therapy," J. Int. Assoc. Physicians AIDS Care, vol. 7, no. SUPPL. 1, pp. 17-21, 2008, doi: 10.1177/1545109708315404

[36] S. Kulkarni et al., "Identifying Perceived Barriers along the HIV Care Continuum: Findings from Providers, Peer Educators, and Observations of Provider-Patient Interactions in Ethiopia HHS Public Access," J Int Assoc Provid AIDS Care, vol. 15, no. 4, pp. 291-300, 2016, doi: 10.1177/2325957415593635.

[37] S. P. Wasti et al., "Factors influencing adherence to antiretroviral treatment in Asian developing countries: A systematic review," Trop. Med. Int. Heal., vol. 17, no. 1, pp. 71-81, 2012, doi: 10.1111/j.1365-3156.2011.02888.x.

[38] R. Horne, J. Weinman, and M. Hankins, "The beliefs about medicines questionnaire: The development and evaluation of a new method for assessing the cognitive representation of medication," Psychol. Heal., vol. 14, no. 1, pp. 1-24, 1999.

[39] S. P. Wasti, P. Simkhada, J. Randall, J. V. Freeman, and E. van Teijlingen, "Factors influencing adherence to antiretroviral treatment in Nepal: A mixed-methods study," PLoS One, vol. 7, no. 5, p. e35547, 2012, doi: 10.1371/journal.pone.0035547.

[40] T. V. Ndou, P. R. Risenga, and M. S. Maputle, "Experiences of HIV positive patients on ARV treatment at the thulamela municipality in the Vhembe district of Limpopo Province, South Africa," J. Antivirals Antiretrovir., vol. 5, no. 5, pp. 123-131, 2013, doi: 10.4172/jaa.1000075.

[41] W. C. Marks David, Murray Michael, Evans Brian, Health Psychology Theory, Research and Practice, First edit. Sage Publication, 2000.

[42] H. M. Do, M. P. Dunne, M. Kato, C. V Pham, and K. V Nguyen, "Factors associated with suboptimal adherence to antiretroviral therapy in Viet Nam: a cross-sectional study using audio computer-assisted self-interview (ACASI).," BMC Infect. Dis., vol. 13, no. 1, 2013, doi: 10.1186/1471-2334-13-154.

[43] M. L. van der Kop et al., "The effect of weekly short message service communication on patient retention in care in the first year after HIV diagnosis: study protocol for a randomised controlled trial (WelTel Retain)," BMJ Open, vol. 3, no. 6, 2013, doi: 10.1136/bmjopen-2013-003155.

[44] W. T. Chen et al., "Engagement with health care providers affects self- efficacy, self-esteem, medication adherence and quality of life in people living with HIV," J. AIDS Clin. Res., vol. 4, no. 11, pp. 256-270, 2013, doi: $10.4172 / 2155-6113.1000256$.

[45] C. A. Inguane et al., "Assessment of linkages from HIV testing to enrolment and retention in HIV care in Central Mozambique,” J. Int. AIDS Soc., vol. 19, no. Suppl 4, pp. 1-8, 2016, doi: 10.7448/IAS.19.5.20846. 